\title{
dspace.vutbr.cz
}

\section{Systematic Design of Pseudo-Differential Frequency Filter}

\author{
SLÁDOK, O.; MILOTA, M.; KOTON, J.; HERENCSÁR, N.
}

Proceedings of the 2019 11th International Congress on Ultra Modern Telecommunications and Control Systems and Workshops (ICUMT), pp. 1-6

elSBN: 978-1-7281-5763-4

ISSN: 2157-023X

DOI: https://doi.org/10.1109/ICUMT48472.2019.8971000

Accepted manuscript

(C2019 IEEE. Personal use of this material is permitted. Permission from IEEE must be obtained for all other uses, in any current or future media, including reprinting/republishing this material for advertising or promotional purposes, creating new collective works, for resale or redistribution to servers or lists, or reuse of any copyrighted component of this work in other works. SLÁDOK, O.; MILOTA, M.; KOTON, J.; HERENCSÁR, N. "Systematic Design of PseudoDifferential Frequency Filter", 2019 11th International Congress on Ultra Modern Telecommunications and Control Systems and Workshops (ICUMT), 2019.

DOl: 10.1109/ICUMT48472.2019.8971000. Final version is available at https://ieeexplore.ieee.org/document/8971000 


\section{Systematic Design of Pseudo-Differential Frequency Filter}

\author{
Ondrej Sladok \\ Department of Telecommunications \\ Brno University of Technology \\ Brno, Czech Republic \\ sladok@phd.feec.vutbr.cz \\ Jaroslav Koton \\ Department of Telecommunications \\ Brno University of Technology \\ Brno, Czech Republic \\ koton@feec.vutbr.cz
}

\author{
Martin Milota \\ Department of Telecommunications \\ Brno University of Technology \\ Brno, Czech Republic \\ xmilot03@stud.feec.vutbr.cz \\ Norbert Herencsar \\ Department of Telecommunications \\ Brno University of Technology \\ Brno, Czech Republic \\ herencsn@feec.vutbr.cz
}

\begin{abstract}
In the paper, the theory of systematic design frequency filters is described. The result is a fourth-order pseudo-differential frequency filter operating in the voltagemode and implemented as a cascade connection of two basic second-order blocks. The filter is able to realize the low-pass filtering functions. The basic block employs three active elements and four passive elements. The filter is characterized by a minimum number of passive elements and high output impedance. Filter analysis examines the magnitude, phase, common-mode rejection ratio signal, and behavior of filters while reducing the recommended voltage. The proper functionality of the filter is verified by simulations and experimental measurements for two different interconnections.
\end{abstract}

Keywords - Pseudo-differential filter, voltage-mode, current conveyor, low-pass, cascade, systematic design

\section{INTRODUCTION}

Frequency filters are now an integral part of most electronic devices. Analog technology remains a little in the background compared to digital technology, which is recording great development. However, we must not forget this technique and therefore this article deals with one area of analog technology - frequency filters. Basic structures are used, the so-called single-ended circuits [1] - [2]. Interest in the design of fully-differential filters is growing, due to high demands for low-voltage and low-power applications [3] [7]. Besides these applications, the emphasis is on improving the active elements or new development of these elements. For the design of new frequency filters, different types of active elements are used. As example, current feedback operational amplifiers (CFOAs) [8], current amplifiers (CAs) [9] - [10], operational transconductance amplifiers (OTAs) [11] - [13], current differential transconductance amplifiers (CDTAs) [14] or elements of the current conveyors (CCs) [15] - [23] can be mentioned. The advantages of fully-differential filters are, for example, enhances the dynamic range, the higher ability to reject power supply noise, reduced the harmonic distortion and reject the common-mode noise signals. On the other hand, the fully-differential filters exhibit generally double circuit complexity compared to single-ended solutions. Singleended circuits have a simpler design but do not have the advantageous properties of fully-differential frequency filters, described above.

Therefore, pseudo-differential frequency filters [24] - [32] were proposed as a possible trade-off between fully- differential and single-ended filters. They provide a simpler structure as single-ended circuits and provide similar features to fully-differential circuits.

In this paper, we deal with the systematic design of pseudo-differential filters working in the voltage-mode. The systematic approach is shown on the description and analysis of a fourth-order low-pass filter being designed as a cascade connection of two basic second-order blocks. The basic building block for systematic design is the secondorder pseudo-differential frequency filter operating in the voltage-mode. The second-order filter uses three active elements - current conveyors and four passive elements. In theory, the input impedance of the filters is infinitely high and hence it is suitable for easy cascading. The cascade interconnection has been verified in two individual solutions, direct and cross interconnection of the two basic blocks. The analysis of the simulation and experimental results show the performance proving that the pseudodifferential frequency filter still features low sensitivity and the high common-mode rejection ratio. Simulation results also compared to experimental measurements prove the functionality of both cascade interconnection and support the theory of pseudo-differential filters.

\section{PSEUDO-DIFFERENTIAL FILTERS}

Pseudo-differential circuits combine the properties of fully-differential and single-ended filters. These structures have differential inputs and differential outputs, but the internal structure, in contrast with fully-differential circuits, remains non-differential. These structures do not contain as many elements as fully-differential circuits, therefore are less complex than fully-differential structures. Still, provide high suppression of the common-mode signal and have lower harmonic distortions. In practice, they are often used in combination with fully-differential filters [28].

As it is evident from the mathematical description (1) and the general view on voltage-mode fully-differential filters (Fig. 1a), only the differential input and differential output signals are assumed it. Therefore, it is generally possible to design differential system using differential input and differential outputs signals, where the inner structure is nondifferential. These structures referred to as pseudodifferential filters (Fig. 1b) 


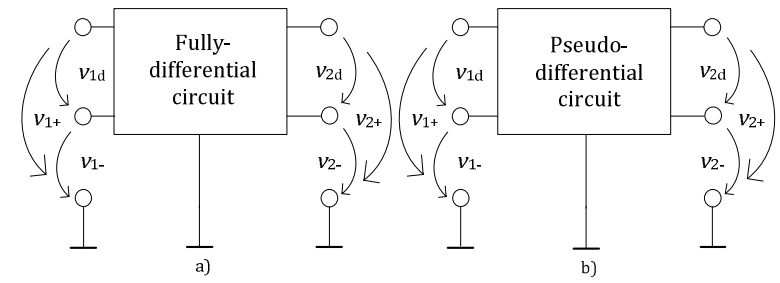

Fig. 1. a) Fully-differential structure, b) Pseudo-differential structure

are described by the following relations:

$$
v_{1 \mathrm{~d}}=v_{1+}-v_{1-}, \quad v_{2 \mathrm{~d}}=v_{2+}-v_{2-}, \quad v_{1 \mathrm{c}}=\frac{v_{1+}-v_{1-}}{2},
$$

being also valid for fully-differential circuits whereas $v_{1 \mathrm{~d}}, v_{2 \mathrm{~d}}$ and $v_{1 \mathrm{c}}$ denote differential input voltage, the differential output voltage, and the common-mode input voltage, respectively. Signal $v_{1 \mathrm{~d}}$ is the difference between the two input signals $v_{1+}$ and $v_{1-}$, while $v_{1 \mathrm{c}}$ is expressing commonmode input signal as the average of the two input signals $v_{1+}$ and $v_{1}$. Taking into consideration (1), then the differential output voltage $v_{2 \mathrm{~d}}$ is defined as follows:

$$
v_{2 \mathrm{~d}}=v_{\mathrm{ld}} \cdot A_{\mathrm{dm}}+v_{1 \mathrm{c}} \cdot A_{\mathrm{cm}}
$$

where $A_{\mathrm{cm}}$ and $A_{\mathrm{dm}}$ are common-mode and differential signal gains, respectively. The rejection of common-mode signal is expressed using $C M R R$ as [33]:

$$
C M R R=20 \log \left(\frac{A_{\mathrm{dm}}}{A_{\mathrm{cm}}}\right),
$$

which determines the ability of an active element or a differential structure to suppress unwanted common-mode input signal that is common to both inputs, to the desired differential input signal. In ideal case $A_{\mathrm{cm}}=0$ and hence CMRR is infinite.

\section{SYSTEMATIC DESIGN OF PSEUDO-DIFFERENTIAL FILTERS}

\section{1) Description of CCII and DDCC}

For the design of pseudo-differential frequency filter operating in voltage-mode the CCII and DDCC active elements have been used, which schematic symbols are shown in Fig. 2.

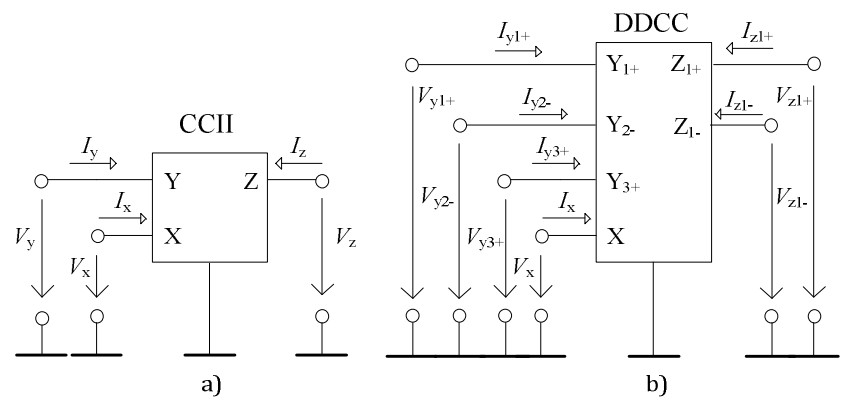

Fig. 2. a) Schematic symbols of CCII, b) Schematic symbols of DDCC

The CCII is a three-terminal building block with one

The research described in this paper was financed by the Research of information and communication systems and their security - FEKT-S-174184. For the research, the infrastructure of the SIX Center was used. high-impedance voltage input $\mathrm{Y}$ and low-impedance current input $\mathrm{X}$, and one high-impedance current output $\mathrm{Z}$.

The relation between terminal currents and voltages is given as [34]:

$$
V_{\mathrm{x}}=V_{\mathrm{y}}, I_{\mathrm{y}}=0, I_{\mathrm{z}}=I_{\mathrm{x}} \text {. }
$$

The DDCC is a six-terminal building block with three high-impedance voltage inputs $\mathrm{Y}_{1+}, \mathrm{Y}_{2-}$ and $\mathrm{Y}_{3+}$, a lowimpedance current input $X$, and two high-impedance current

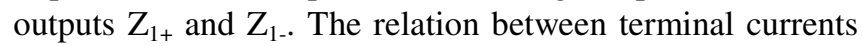
and voltages is given as [34]:

$$
\begin{gathered}
V_{\mathrm{x}}=V_{\mathrm{y} 1+}-V_{\mathrm{y} 2-}+V_{\mathrm{y} 3+}, I_{\mathrm{y} 1+}=I_{\mathrm{y} 2-}=I_{\mathrm{y} 3+}=0, \\
I_{\mathrm{z} 1+}=I_{\mathrm{x}}, I_{\mathrm{z} 1-}=-I_{\mathrm{x}} .
\end{gathered}
$$

2) Second-order pseudo-differential frequency filter

The basic block for systematic analysis is the second order pseudo-differential frequency filter. The circuit is realized using three active elements two DDCC and one CCII and four passive elements, where are all grounded. The differential input signal is directly led to the $\mathrm{Y}_{1+}$ and $\mathrm{Y}_{2-}$ terminals of DDCC1, Cascade connection is possible due to the high input impedance of the circuit and next advantage is the capacitors are not connected to terminals $\mathrm{X}$, which in practice would result in additional parasitic poles.

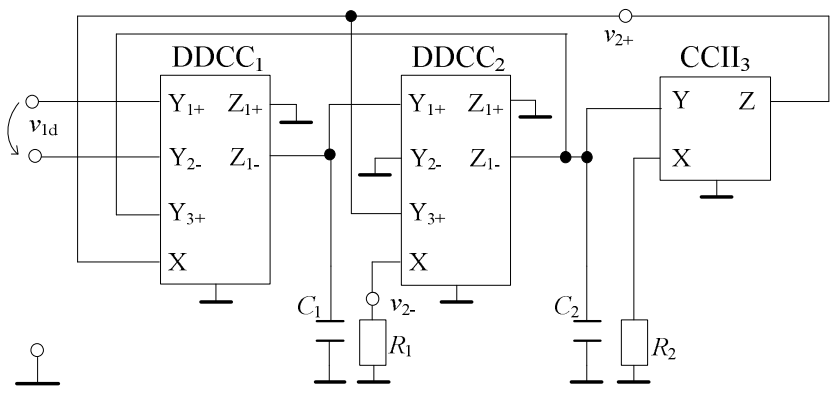

Fig. 3. Proposed second-order pseudo-differential frequency filter

Assuming the input and output nodes as denoted in Fig. 3, the structure allows realizing pseudo-differential secondorder low-pass response since for the positive and negative output voltage $v_{2+}$ and $v_{2-}$ it holds:

$$
\begin{aligned}
& v_{2+}=\frac{s^{2} C_{1} C_{2} R_{1} R_{2}+1}{s^{2} C_{1} C_{2} R_{1} R_{2}+s C_{1} R_{2}+1} \cdot v_{1 \mathrm{~d}}+0 \cdot v_{1 \mathrm{c}}, \\
& v_{2-}=\frac{s^{2} C_{1} C_{2} R_{1} R_{2}}{s^{2} C_{1} C_{2} R_{1} R_{2}+s C_{1} R_{2}+1} \cdot v_{1 \mathrm{~d}}+0 \cdot v_{1 \mathrm{c}},
\end{aligned}
$$

and then for pseudo-differential-output voltage $v_{2 \mathrm{~d}}$ it can be written:

$$
v_{2 \mathrm{~d}}=\frac{1}{s^{2} C_{1} C_{2} R_{1} R_{2}+s C_{1} R_{2}+1} \cdot v_{1 \mathrm{~d}}+0 \cdot v_{1 \mathrm{c}},
$$

if ideal active elements are considered. Comparing (6c) with 
(2) we get differential gain:

$$
A_{\mathrm{dm}}=\frac{1}{s^{2} C_{1} C_{2} R_{1} R_{2}+s C_{1} R_{2}+1},
$$

\section{3) Fourth-order pseudo-differential frequency filter}

The proposed fourth-order pseudo-differential frequency filter was realized as the cascade connection of basic second-order blocks from Fig. 3 .

The functionality of the filter was verified by two different connections of the basic blocks, i.e. direct and cross (Fig. 4).

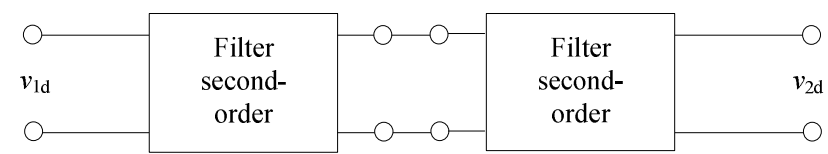

a)

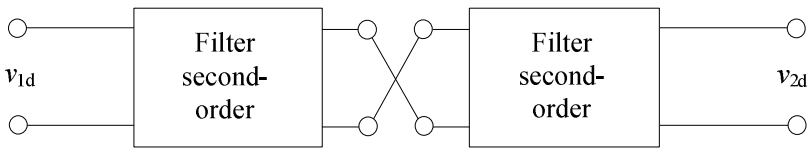

b)

Fig. 4. Cascade connections a) Direct b) Cross

Direct and cross systematic designs in Fig. 4. have been subjected to simulations and experimental measurements. For both cascade connections, the low-pass response of the fourth-order pseudo-differential frequency filter can be described as:

$$
A_{\mathrm{dm}}=\frac{1}{s^{4}\left(b_{4}\right)+s^{3}\left(b_{3}\right)+s^{2}\left(b_{2}\right)+s\left(b_{1}\right)+b_{0}}
$$

where

$$
\begin{aligned}
& b_{4}=\left(C_{11} C_{12} C_{21} C_{22} R_{11} R_{12} R_{21} R_{22}\right), \\
& b_{3}=\left(C_{12} C_{21} C_{22} R_{12} R_{21} R_{22}+C_{11} C_{12} C_{21} R_{11} R_{12} R_{21}\right), \\
& b_{2}=\left(C_{11} C_{12} R_{11} R_{12}+C_{21} C_{22} R_{21} R_{22}+C_{12} C_{21} R_{12} R_{21}\right), \\
& b_{1}=\left(C_{12} R_{12}+C_{21} R_{21}\right), b_{0}=1 .
\end{aligned}
$$

\section{SimUlations AND EXPERIMENTAL MEASUREMENTS}

The behavior and performance of the proposed pseudodifferential filters have been evaluated by simulations. Furthermore, the behavior has been verified experimental measurements, was made for recommended supply voltages $( \pm 1,65 \mathrm{~V})$ according to [38] and for reduced supply voltages $( \pm 1,50 \mathrm{~V})$. The reduced supply voltage is a 90 percent value selected from the recommended supply voltage value. To realize the active elements CCII and DDCC, the universal current conveyor UCC-N1B 0520 [38] was used. Butterworth coefficients were used for the calculation of the 2nd- and 4th-order filters and pole-frequency $\mathrm{f}_{0}=50 \mathrm{kHz}$.

\section{1) Second-order pseudo-differential frequency filter}

For simulations and experimental measurements the values of capacitors $C_{1}=C_{2}=1 \mathrm{nF}$ were selected and the values of resistors were determined as $R_{1}=2250 \Omega$ and $R_{2}=$ $4500 \Omega$ to obtain the pole-frequency $50 \mathrm{kHz}$ and quality factor $Q=0.707$. The obtained frequency responses of the pseudo-differential second-order filter operating in voltagemode are shown in Fig. 5.

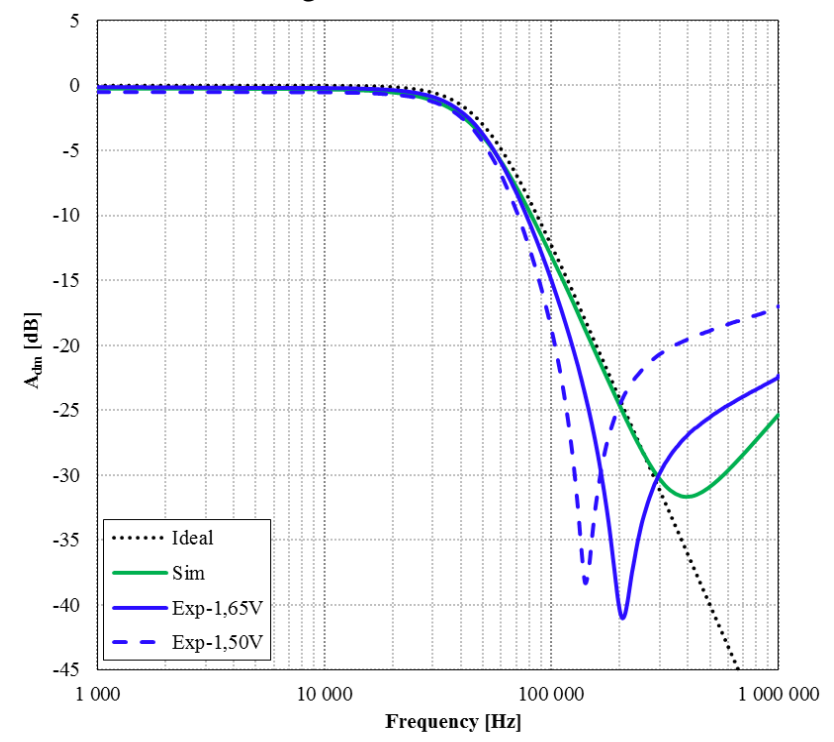

a)

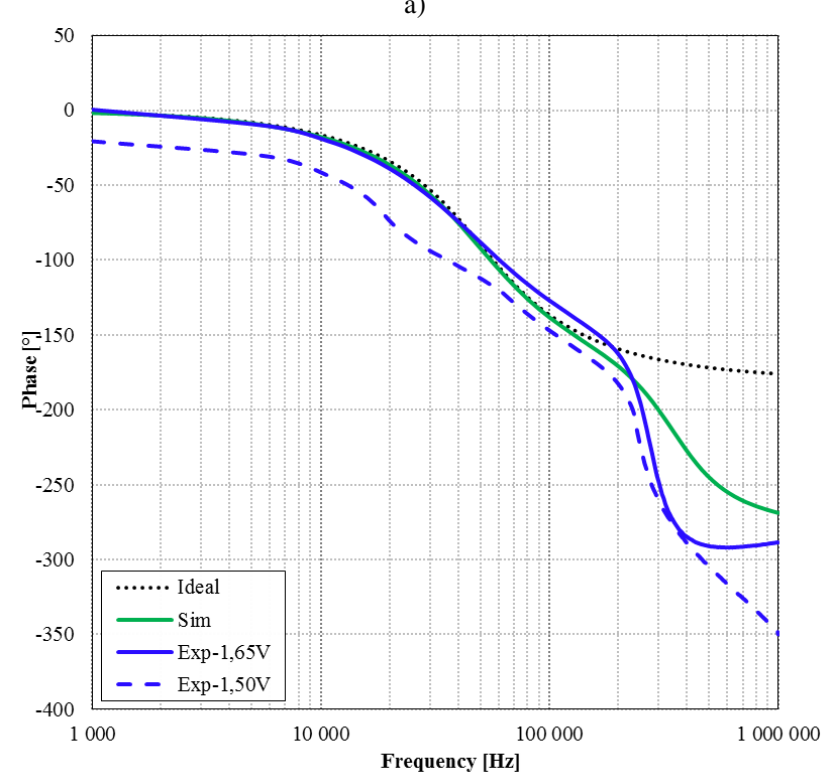

b)

Fig. 5. Magnitude and phase characteristics of the low-pass secondorder filter

From both the simulations and experimental measurements shown in Fig. 5 we can claim that they are in very good agreement with the theory. The worse result shows by measuring with reduced supply voltages but that is the expected behavior. Better result of the magnitude characteristic shows the experiment $( \pm 1,65 \mathrm{~V})$, where the response is closer to the simulation. The real properties of the prototype cause a parasitic zero, which manifests around a frequency of approximately $200 \mathrm{kHz}$ for simulations and experimental measurements.

The phase characteristics for both supply voltage were achieving nearly ideal characteristics. 


\section{2) Fourth-order pseudo-differential frequency filter}

The fourth-order pseudo-differential filter operating in the voltage mode was designed as a cascade connection of the basic second-order solution from Fig. 3. For simulations and measuring, Butterworth approximation was used. The values of capacitors $C_{11}=C_{12}=C_{21}=C_{22}=1 n F$ were selected and the resistor values $R_{11}=1700 \Omega, R_{12}=5900 \Omega, R_{21}=$ $2400 \Omega, \mathrm{R}_{22}=4200 \Omega$ were calculated resulting in $50 \mathrm{kHz}$ pole-frequency. The plots in Fig. 6. show magnitude and phase characteristics.

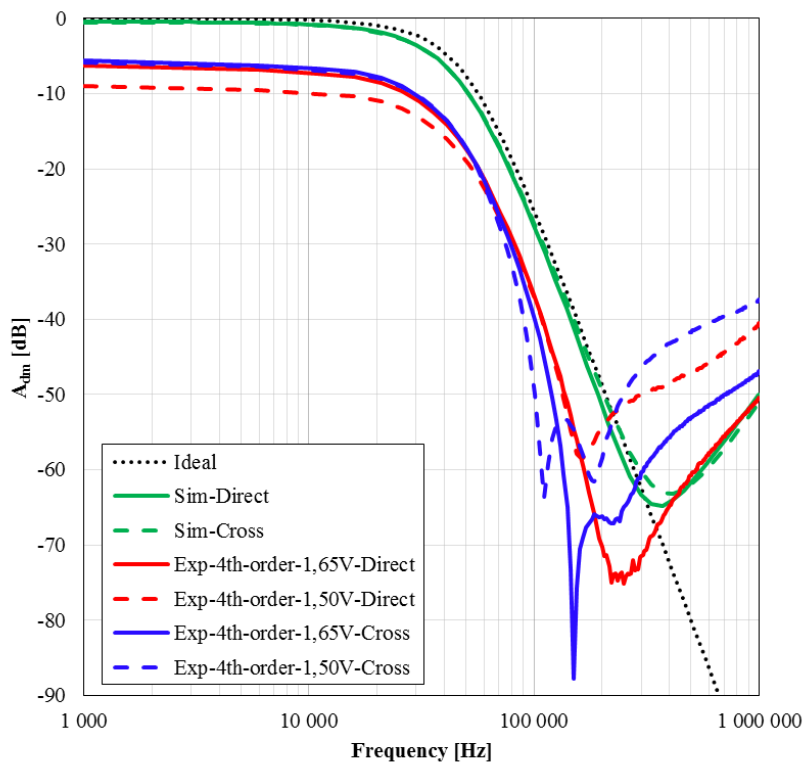

a)

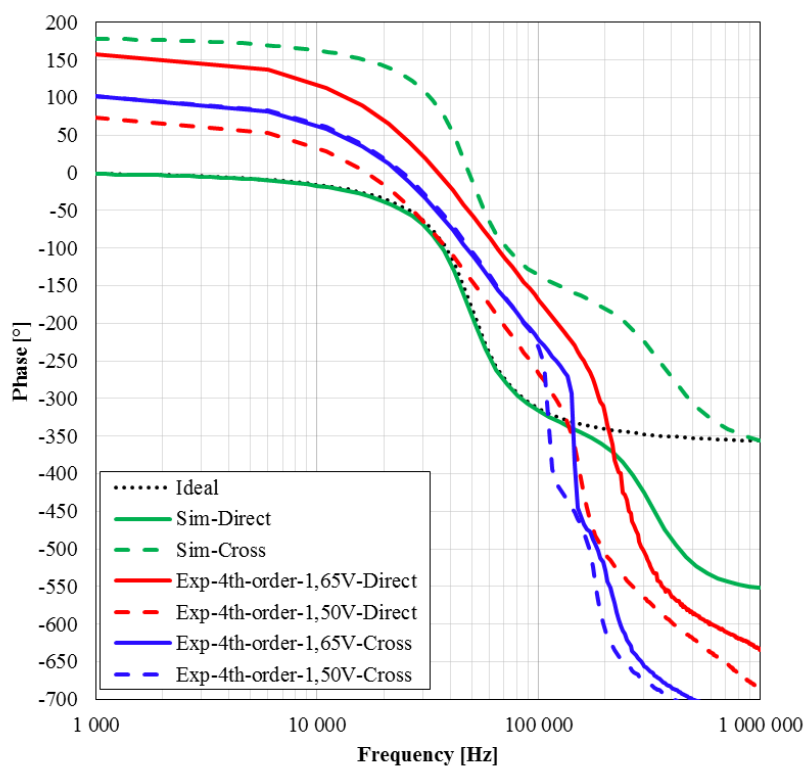

b)

Fig. 6. Magnitude and phase characteristics of the low-pass fourthorder filter

From the magnitude characteristics, it is clear that all experimental measurements are suppressed approximately $6 \mathrm{~dB}$. The real properties of the integrated circuit at approximately $200 \mathrm{kHz}$ were also reflected in the fourthorder filter. However, it is necessary to say that all responses copy to simulations characteristic.

From the phase characteristics in Fig. 6b) exhibit the greatest differences from all the results. Better result of the phase characteristic shows the experiment $( \pm 1,65 \mathrm{~V})$ connection cross, but it has to be said that to the ideal transfer it is only approaching. The parasitic properties are caused by real properties of the active elements emulated by the UCC_N1B [38] simulation active elements.

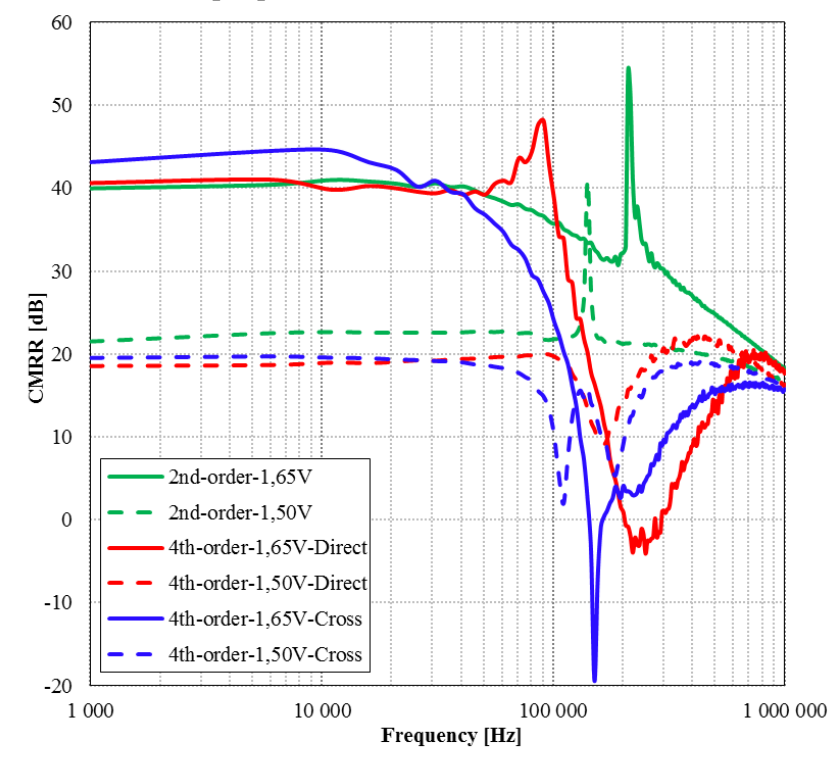

Fig. 7. The common-mode rejection ratio (CMRR) obtained by experimental measurements.

For both, pseudo-differential frequency filters (2nd-order and 4th-order) were measured the results and are shown in Fig. 7. The reached value of CMRR is approximately $40 \mathrm{~dB}$ and is constantly up to $200 \mathrm{kHz}$. The theoretical value of the common-mode rejection ratio is $43 \mathrm{~dB}$ according to [28]. Drop around the frequency of $200 \mathrm{kHz}$ is again a manifestation of real properties UCC-N1B 520 that create a parasitic zero. This phenomenon is already visible in the magnitude characteristics of Fig. 5a) and Fig. 6a), then the CMRR continues. When the supply voltage is reduced, the CMRR value is approximately $20 \mathrm{~dB}$. Therefore, the optimum supply voltage must be supplied for functionality.

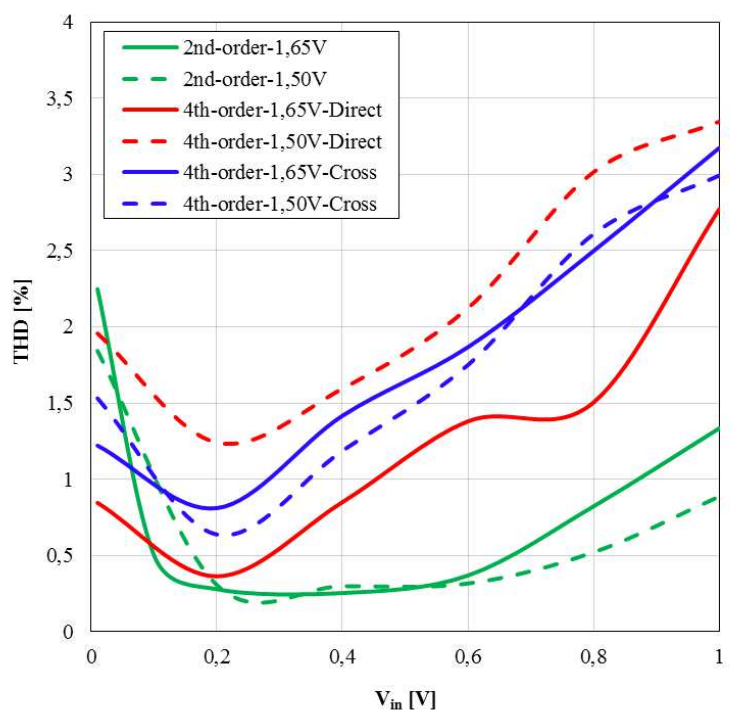

Fig. 8. Total harmonic distortion (THD) obtained by experimental measurements.

Next to CMRR the THD (total harmonic distortion) has 
been measured. Evaluating THD, the first five major harmonic components were considered, whereas the $1 \mathrm{kHz}$ input signal has been applied to $V_{\text {in. }}$. As it can be seen in Fig. 8. for all experimental measurements, for input signal amplitude below $0.6 \mathrm{~V}$ the total harmonic distortion is less than $2 \%$. For higher input signal amplitudes THD starts to decrease as the active elements start to reach their voltage and current saturation levels [38].

Simulations in PSpice evaluated the behavior and performance of the proposed pseudo-differential filters. They were then subjected to experimental measurements using an Agilent 4392A Network/Spectrum Analyzer. Other integrated circuits were used for experimental measurements. The AD8476 circuit [35] was used to obtain the input differential signal. Furthermore, two AD8271 summation amplifiers were used for the common-mode rejection ratio [36]. The output differential signal was converted to a single-ended signal using AD8429 [37]. The complete structure of the experimental measurement can be seen in Fig. 9., where is a block diagram of a pseudodifference filter together with S/D and D/S converters and Fig. 10, where is the original design of the fourth-order pseudo-difference filter again together with the converters.

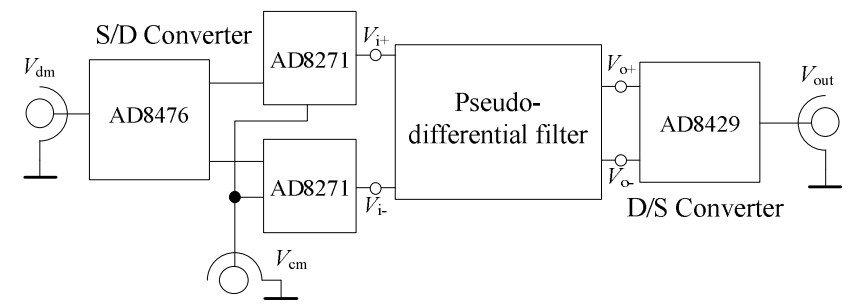

Fig. 9. The block diagram of the experimental measurement

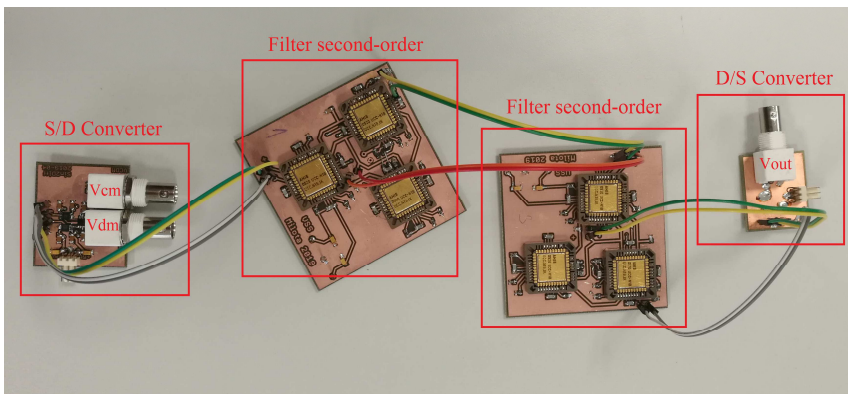

Fig. 10. The original design of the fourth-order pseudo-differential frequency filter

\section{CONCLUSION}

In this paper, a new systematic design of fourth-order pseudo-differential frequency filter operating in voltage mode is presented. The theory of pseudo-differential filters was investigated, where the possibility of designing higherorder filters was analyzed. Using the basic block - secondorder pseudo-differential frequency filter connected in cascade, the fourth-order pseudo-differential filter operating in the voltage-mode was designed. The functionality and performance of the filter have been verified by both simulations and experimental measurements. Both the simulation and experimental measurements agree well with the theoretical analyses. The total harmonic distortion is less than $2 \%$ for the amplitude of the input signal below $0,6 \mathrm{~V}$. The value of CMRR reached by measurements is approx. 40 $\mathrm{dB}$. This article more confirms the theory of pseudodifferential frequency filters. This is proved by achieving generally the same results in the frequency response of the filter for direct and cross interconnections of the partial second-order blocks. Based on the presented results, it was shown that the theory of pseudo-differential filter is valid and fully functional.

\section{REFERENCES}

[1] W.-K. Chen, The circuits and fiters handbook, (3rd edition, CRC Press, 2003), p. 2961.

[2] L. Langhammer, J. Jerabek, J. Polak, P. Cika. Single-Ended and FullyDifferential Current-Input Current-Output Universal Frequency Filter with Transconductace and Transresistance Amplifiers, WSEAS TRANSACTIONS on CIRCUITS and SYSTEMS, Canary Islands, Spain, 2015, pp. 56-67.

[3] P. Beg, I. A. Khan, S. Maheshwari, and M. A. Siddiqui, Digi-tally Programmable Fully Differential Filter, Radioengineering 20 (2011) 917-925.)

[4] N. Herencsar, J. Jerabek, J. Koton, K. Vrba, S. Minaei, I. C. Goknar, Pole Frequency and Pass-Band Gain Tunable Novel Fully-Differential Current-Mode All-Pass Filter, IEEE Int. Symposium on Circuits and Systems (ISCAS), May 2015.

[5] J. Dvorak, J. Jerabek, L. Langhammer, S. Kapoulea, C. Psychalinos. (2018). Design of Fully-Differential Frequency Filter with FractionalOrder Elements. DOI: 10.1109/TSP.2018.8441259.

[6] L. Langhammer, R. Sotner, J. Dvorak, J. Jerabek and M. Zapletal, Fully-Differential Universal Frequency Filter with Dual-Parameter Control of the Pole Frequency and Quality Factor, 2018 IEEE International Symposium on Circuits and Systems (ISCAS), Florence, 2018, pp. 1-5.doi: 10.1109/ISCAS.2018.8351005

[7] J. Jerabek, K. Vrba. Design of Fully Differential Filters with Basic Active Elements Working in the Current Mode, Elektrorevue, vol. 12, no.6, pp. 87-1 - 87-5, 2010.

[8] G. Tsirimokou, S. Koumousi and C. Psychalinos, Design of Fractional-Order Filters Using Current Feedback Operational Amplifiers, In Proc. 3rd Pan-Hellenic conference on electronics and telecommunications (PACET 2015), 2015.

[9] J. Jerabek, R. Sotner and K. Vrba, General Current-Mode Filtering Structure with Controllable Current Active Elements, In Proc. 36th international conference on telecommunications and signal processing (TSP 2013), 2013, pp. 402-406.

[10] J. Polak, J. Jerabek, L. Langhammer, R. Sotner, J. Dvorak and D. Panek, Digitally Controllable Current Amplifier and Current Conveyors in Practical Application of Controllable Frequency Filter, Journal of Electrical Engineering, 2016, vol. 67, pp. 261-266.

[11] J. Jerabek, R. Sotner and K. Vrba, Electronically Adjustable TripleInput Single-Output Filter with Voltage Differencing Transconductance Amplifier, Revue Roumaine des Sciences Techniques - Serie Electrotechnique et 'Energ'etique, ' 2015, vol. 59, pp. 163-172.

[12] R. Sotner, N. Herencsar, J. Jerabek, R. Prokop, A. Kartci, T. Dostaland K. Vrba, Z-Copy Controlled-Gain Voltage Differencing Current Conveyor: Advanced Possibilities in Direct Electronic Control of First-Order Filter, Elektronika ir elektrotechnika, 2014, vol. 20, pp. 77-83.

[13] D. Agrawal, S. Maheshwari, Cascadable current mode instrumentation amplifier, AEU - International Journal of Electronics and Communications, Volume 94, 2018, Pages 91-101, ISSN 14348411, https://doi.org/10.1016/j.aeue.2018.06.038.

[14] M. A. Eldeeb, Y. H. Ghallab, Y. Ismail, H. Elghitani, Low-Voltage Subthreshold CMOS Current Mode Circuits: Design and Applications, AEU - International Journal of Electronics and Communications, Volume 82, 2017, Pages 251-264, ISSN 14348411, https://doi.org/10.1016/j.aeue.2017.08.049.

[15] P. Farago, L. Festila, S. Hintea, G. Csipkes, D. Csipkes, P. Soser, A Transistor-Level Reconfigurable Circuit for Rapid Transconductor Design and Testing, Elektronika ir Elektrotechnika, vol. 117, no. 1, pp. 99-104, 2012 [Online]. 
[16] Ch. Hua-Pin, H. Yuh-Shyan, K. Yi-Tsen, A Systematic Realization of Third-Order Quadrature Oscillator with Controllable Amplitude, AEU - International Journal of Electronics and Communications, Volume 79 , https://doi.org/10.1016/j.aeue.2017.05.039. 2017, Pages 64-73, ISSN 1434-8411,

[17] J. Jerabek and K. Vrba, SIMO Type Low-Input and High-Output Impedance Current-Mode Universal Filter Employing Three Universal Current Conveyors, International Journal of Electronics and Communications (AEU), 2010, vol. 64, pp. 588-593.

[18] F. Khateb, D. Kubanek, G. Tsirimokou and C. Psychalinos, Fractional-Order Filters Based on Low-Voltage DDCCs. Microelectronics Journal, 2016, 50, pp. 50-59.

[19] E. Yuce. A Single-Input Multiple-Output Voltage-Mode SecondOrder Universal Filter Using Only Grounded Passive Components. Indian Journal of Engineering \& Materials Sciences. Vol. 24, April 2017, pp. 97-106.

[20] H. Chen, Current-mode dual-output ICCII-based tunable universal biquadratic filter with low-input and high output impedances", International Journal of Circuit Theory and Applications, vol. 42, Issue 4, pp. 376-393, 2012. [Online]. Available: http://dx.doi.org/10.1002/cta.1858

[21] J.-W.Horng, W.-Y. Chiu, T.-Y. Teng, C.-H. Lin, N. Herencsar, J. Koton, Cascadable Voltage- Mode Three Inputs Universal Biquad Using DVCCs. In Proc. of 2014 14th International Symposium on Communications and Information Technologies (ISCIT). 2014. s. 299-303. ISBN: 978-1-4799-4416- 3.

[22] F. Kacar, S. Menekay, Realization of A CMOS Current Differencing Buffer Amplifier and Its Filter Application, in Proc. The 7th International Conference Electrical and Electronics Engineering (ELECO), Bursa, Turkey, 2011, pp. 337-340.

[23] B. Metin, N. Herencsar, J. Koton, E. Arslan. All- Pass Filter Application Using Electronically Tunable DDCC. In Proceedings of the 24th International Conference Radioelektronika 2014. Bratislava: 2014. s. 1-4. ISBN: 978-1-4799-3714- 1.

[24] O. Sladok, L. Velic, J. Koton, N. Herencsar. Pseudo-differential Filter Operating In Current Mode. In 2018 10th International Congress on Ultra Modern Telecommunications and Control Systems. International Congress on Ultra Modern Telecommunications and Control Systems and Workshops. IEEE, 2018. s. 252-257. ISBN: 978-1-5386-9361-2. ISSN: 2157-023X.

[25] N. Herencsar, R. Sotner, A. Kartci and K. Vrba, A Novel PseudoDifferential Integer/ Fractional-Order Voltage-Mode All-Pass Filter, 2018 IEEE International Symposium on Circuits and Systems (ISCAS), $\quad$ Florence, $\quad 2018, \quad$ pp. doi: 10.1109/ISCAS.2018.8351520
[26] O. Sladok. Pseudo-Differential High-Order Frequency Filter. In Proc. 25nd conf. Student EEICT. 2019. s. 764-768. ISBN 978-80-2145735-5.

[27] M. Milota. Pseudo-Differential Frequency Filter Using Conveyors. In Proc. 25nd conf. Student EEICT. 2019. s. 66-69. ISBN 978-80214-5735-5.

[28] J. Koton, N. Herencsar, O. Sladok, J. Horng, Pseudo-Differential Second-Order Band Reject Filter Using Current Conveyors, AEU International Journal of Electronics and Communications, vol. 70, no. 6, pp. 814-821, 2016. [Online]. Available: http://dx.doi.org/10.1016/j.aeue.2016.03.009

[29] J. Koton, N. Herencsar, J.-W. Horng, Differential Second-Order Voltage-Mode All-Pass Filter Using Current Conveyors, Elektronika ir Elektrotechnika, vol. 22, no. 5, pp. 52-57, 2016. [Online]. Available: http://dx.doi.org/10.5755/j01.eie.22.5.16344

[30] O. Sladok, J. Koton, N. Herencsar. Universal Pseudo-Differential Filter Using DDCC and DVCCs. Elektronika Ir Elektrotechnika, 2017, vol. 23, no. 6, p. 46-52. ISSN: 1392-1215. [Online]. Available: http://dx.doi.org/10.5755/j01.eie.23.6.19694

[31] O. Sladok. Pseudo-Differential Filter Using DDCC. In Proc. 22nd conf. Student EEICT. 2016. s. 147-149. ISBN: 978-80-214-5350- 0.

[32] R. Sotner, N. Herencsar, J. Jerabek, A. Kartci, J. Koton, T. Dostal.. Pseudo-Differential Filter Design Using Novel Adjustable Floating Inductance Simulator with Electronically Controllable Current Conveyors. Elektronika Ir Elektrotechnika, 2017, vol. 23, no. 2, p. 3135. ISSN: 1392-1215.

[33] U. Tietze, Ch. Schenk, E. Gamm, Electronic Circuits: Handbook for Design and Application. Berlin: Springer. 2008, p. 1543. [Online]. Available: http://dx.doi.org/10.1007/978-3-540-78655-9

[34] R. Senani, D. R. Bhaskar, A. K. Singh, Current Conveyors: Variant,Applications and Hardware Implementations. Switzerland: Springer-Verlag, $2015 . \quad$ [Online]. Available: http://dx.doi.org/10.1007/978-3-319-08684-2

[35] Datasheet AD 8476. Low power, unity gain, fully differential amplifier and ADCdriver, analog devices, rev. B.; 2012.

[36] Datasheet AD 8271. Programmable gain precision difference amplifier, analogdevices, rev. 0; 2009.

[37] Datasheet AD 8429. $1 \mathrm{nV} / \sqrt{ } \mathrm{Hz}$ low noise instrumentation amplifier, analogdevices, rev. 0; 2011.

[38] Datasheet UCC-N1B 0520. Universal current conveyor (UCC) and second-generation current conveyor $(\mathrm{CCII}+/-)$, rev. 1. Brno University of Technology, On Semiconductor Ltd.; 2012. 